\title{
Optimized Traffic Signal Control System at Traffic Intersections Using Vanet
}

\author{
V.Hemakumar ${ }^{1}$, H.Nazini ${ }^{2}$ \\ ${ }^{1}$ (Computer Science Engineering, Tagore Engineering College/ AnnaUniversity, India) \\ ${ }^{2}$ (Computer Science Engineering, Tagore Engineering College/ AnnaUniversity, India)
}

\begin{abstract}
Traditional Automated traffic signal control systems normally schedule the vehicles at intersection in a pre timed slot manner. This pre-timed controller approach fails to minimize the waiting time of vehicles at the traffic intersection as it doesn't consider the arrival time of vehicles. To overcome this problem an adaptive and intelligent traffic control system is proposed in such a way that a traffic signal controller with wireless radio installed at the intersection and it is considered as an infrastructure. All the vehicles are equipped with onboard location, speed sensors and a wireless radio to communicate with the infrastructure thereby VANET is formed. Once the vehicles enter into the boundary of traffic area, they broadcast their positional information as data packet with their encapsulated ID in it. The controller at the intersection receives the transmitted packets from all the legs of intersection and then stores it in a temporary log file. Now the controller runs Platooning algorithm to group the vehicles approximately in equal size of platoons. The platoons are formed on the basis of data disseminated by the vehicles. Then the controller runs Oldest Job First algorithm which treats platoons as jobs. The algorithm schedules jobs in conflict free manner and ensures all the jobs utilize equal processing time i.e the vehicles of each platoons cross the intersection at equal delays. The proposed approach is evaluated under various traffic volumes and the performance is analyzed.
\end{abstract}

Keywords Conflict graphs, online job scheduling, traffic signal control, vehicular ad hoc network (VANET) simulation, vehicle-actuated traffic signal control, Webster's algorithm.

\section{INTRODUCTION}

In this paper, we examine the possibility of deploying an intelligent and real-time adaptive traffic signal controller, which receives information from vehicles, such as the vehicle's position and speed, and then utilizes this information to optimize the traffic signal scheduling at the intersection. This approach is enabled by onboard sensors in vehicles and standard wireless communication protocols specifically for vehicular applications. For example, all vehicles are already equipped with a speed sensor. In addition, new vehicles are increasingly being equipped with Global Positioning System (GPS) units that can provide location information with accuracy of a few meters [25].

Furthermore, vehicles can use wireless communications for vehicle-to-vehicle (V2V) or vehicle-toinfrastructure (V2I) communications, as described in the dedi- cated short-range communications/wireless access in vehicular environments standards operating in the spectral range of 5.85-5.95 GHz [19]. We refer to the transient mesh networks formed via V2V or V2I communication links as vehicular ad hoc networks (VANETs).

Intelligent traffic signal control has been extensively studied in the literature [9], [25], [28].Current methods of implementing intelligent traffic signal control include roadside sensors, such as loop detectors and traffic monitoring cameras. Loop detectors can only detect the presence or absence of vehicles [15], [16], which is a serious limitation.

\section{MotiVATION}

The main motivation for vehicular communication systems is safety and eliminating the excessive cost of traffic collisions. According to World Health Organizations (WHO), road accidents annually cause approximately 1.2 million deaths worldwide; one fourth of all deaths caused by injury. Also about 50 million persons are injured in traffic accidents. If preventive measures are not taken road death is likely to become the third-leading cause of death in 2020 from ninth place in 1990. A study from the American Automobile Association (AAA) concluded that car crashes cost the United States $\$ 300$ billion per year.However the deaths caused by car crashes are in principle avoidable. US Department of Transport states that 21,000 of the annual 43,000 road accident deaths in the US are caused by roadway departures and intersection-related incidents. This number can be significantly lowered by deploying local warning systems through vehicular communications. Departing vehicles can inform other vehicles that they intend to depart the highway and arriving cars at intersections can send warning messages to other cars traversing that intersection. Although the main advantage of vehicular networks is safety improvements, there are several other benefits. Vehicular networks can help in 
avoiding congestion and finding better routes by processing real time data. This in return saves both time and fuel and has significant economic advantages.

\section{DEVELOPMENT}

Vehicular communications is mainly motivated by the desire to implement Intelligent Transport Systems (ITS) because of their key benefits in safety and traveling ease. Several ITS institutions operate around the world to bring ITS concepts to real world. In the United States one of the main players is U.S. Department of Transportation (USDoT). The federal DoT promotes ITS through investment in potentially high payoff initiatives. One of these major initiatives, Vehicle Infrastructure Integration (VII), seeks to increase safety by providing vehicle to vehicle and vehicle to roadside units communications through Dedicated Short Range Communications (DSRC).

\section{VANET APPLICATIONS}

The speed and location information on vehicles that can be disseminated to the traffic signal controller using VANETs [10] are both spatially and temporally fine-grained. Such precise per- vehicle speed and location information can enable additional capabilities such as being able to predict the time instance when vehicles will reach the stop line of the intersection.

This is in comparison with roadside sensors such as loop detectors that can only detect the presence or absence of vehicles and, at best estimate, the size of vehicle queues. Furthermore, it is cheaper to equip vehicles with wireless devices than to install roadside equipment [25].

\section{OUR CONTRIBUTION}

In this paper, we present an algorithm, which we call the oldest arrival first (OAF) algorithm, that makes use of the per-vehicle real time position and speed data to do vehicular traffic scheduling at an isolated traffic intersection with the objective of minimizing delays at the intersection. This simple algorithm leads to a near optimal (delay minimizing) schedule that we analyze by reducing the traffic scheduling problem to a job scheduling problem, with conflicts, on processors. The scheduling algorithm captures the conflicts among opposing vehicular traffic with a conflict graph [9], and the objective of the algorithm is to minimize the latency values of the jobs. If the condition that all jobs require equal processing time is enforced, we can show that the OAF algorithm becomes the oldest job first (OJF) algorithm in the job scheduling domain with conflicts between jobs and the objective of minimizing job latency values. We present a 2-competitive (with respect to joblatencies) online algorithm that does nonclairvoyant scheduling [27] with conflicts of the jobs on the processors and then prove a stronger result that the best possible nonclairvoyant scheduling with conflicts algorithm is 2-competitive. We leverage a VANET to implement the OJF algorithm.

An important requirement for the OJF algorithm is that all jobs require equal processing time. We give an algorithm that uses the VANET to divide up the approaching vehicular traffic into platoons that can be treated as jobs in the job scheduling with conflicts. The traffic signal controller can then use the conflict- free schedule from the OJF algorithm to schedule platoons of vehicles in a safe conflict-free manner. This two-phase approach, where we first use the platooning algorithm to divide up the traffic into platoons and then treat each platoon as an equal-sized job and then apply the OJF algorithm on the jobs to generate a conflict-free schedule, leads to what we call the OAF algorithm. To ascertain the performance of the algorithm, we choose the average delay per vehicle that has passed through the intersection as the measure of effectiveness. We compare the performance of the OAF algorithm against an vehicle-actuated traffic signal controller, Webster's algorithm, and a fixed-time algorithm. The vehicle-actuated algorithm and Webster's algo- rithm are well-known traffic algorithms [9] that traditionally utilize fixed road-based sensors such as loop detectors.

\section{TRAFFIC LIGHT SCHEDULING ID REDUCED TO JOB SCHEDULING (OJF ALGORITHM)}

Here, we propose a method to reduce traffic signal control problem to the problem of scheduling jobs on processors, and we propose an online job scheduling algorithm called the OJF algorithm. This is phase two of the OAF two-phase traffic signal control algorithm. 


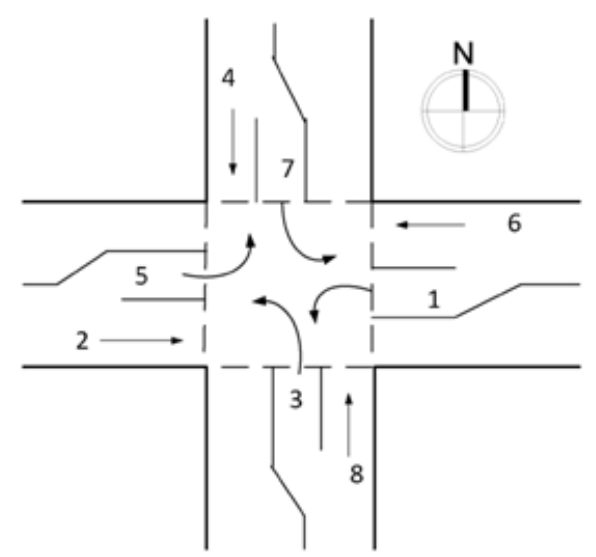

Fig. 1. Typical four-leg intersection showing the different movements on the approaches.

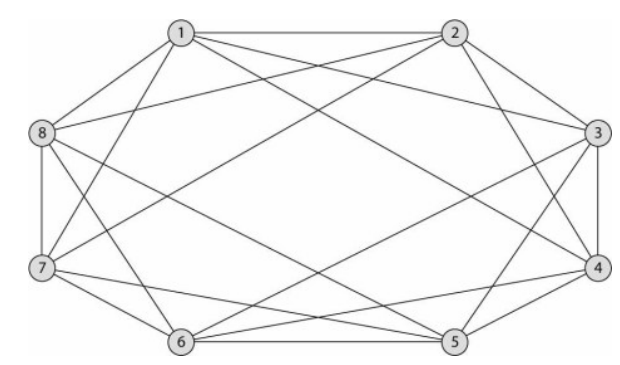

Fig. 2. Conflict graph for the intersection in Fig. 1.

Fig. 1 shows a typical four-leg intersection with eight traffic movements numbered 1-8. This type of intersection is the most common and well-studied type [7], [8]. There are conflicts among some of these movements. For example, traffic move-ments 1 and 2 cannot simultaneously occur. We can reduce the problem of traffic signal control to scheduling of jobs on a processor, where a job is a platoon of one or more vehicles. We classify jobs as follows. A job is of type $i$ if and only if the platoon of vehicles that it represents is part of traffic movement $i$. A pair of jobs of type $i$ and $j$ are said to be in conflict if the traffic movements $i$ and $j$ are in conflict; hence, jobs of type $i$ and $j$ cannot be scheduled to be simultaneously processed. For the intersection in Fig. 1, we can build a conflict graph $\mathrm{G}(\mathrm{V}, \mathrm{E})$, where $\mathrm{V}$ is a set of vertices, and $\mathrm{E}$ is a set of arcs. There is a vertex for each job type, i.e., for each job type $\exists$ vertex $i \in \mathrm{V}$.

The arc set $E$ is constructed as follows. If jobs of type $i, j$ are in conflict (and cannot be scheduled simultaneously), then there exists an arc (i, j) in E. E does not contain any other arc, and V does not contain any other vertex. The conflict graph for the four-leg intersection in Fig. 1 is shown in Fig. 2. Conflict graphs have been studied by traffic engineers to build safe traffic signal control plans. In [9], methods of developing safe signal control plans are shown for more complicated traffic intersections. We will assume that jobs are of equal size, and each job $\mathrm{i}$ of type $\mathrm{j}$ has an arrival time aji, which would correspond to the instance of time when the first vehicle of platoon $i$ arrives at the stop line in movement $\mathrm{j}$. We will assume that time is divided into slots, and since all jobs are equal, without loss of generality, we can assume that all jobs need 1 unit of time to complete. Thus, if a job is scheduled at time $t$, it will complete at time $t+1$. The ability to divide the oncoming traffic into platoons that require approximately equal amount of GREEN time (the green time represents the amount of processing time required) is achieved using a VANET. At the beginning of time unit $t$, jobs of any type $\mathrm{j}$ can arrive, and we can think of them as arriving at vertex $\mathrm{j}$ in $\mathrm{G}$. A group of vertices is chosen that do not conflict, and a job from each of these is scheduled in time t. Now, our objective would be to minimize the maximum latency over all jobs. For a particular job aji, the latency is di - aji - 1, where di is the time unit at the beginning of which job $i$ has disappeared (completed), and aji is the time unit at the beginning of which job $i$ of type $\mathrm{j}$ arrived.

Therefore, the objective is simply to minimize the maximum latency. In the context of vehicular traffic, minimizing maximum latency is equivalent to minimizing the maximum time that any vehicle spends at rest at an intersection waiting for green light. An important simplification that we make here is that all jobs need equal service time. 


\section{A. 2-COMPetitive Algorithm for Job SCHEduling}

Having made the reduction from vehicular traffic scheduling to job scheduling with conflicts, we present a 2-competitive algorithm that minimizes latency for each job that we call the OJF scheduling algorithm. In addition, it turns out that, under the assumption of no future knowledge, this is the best possible online algorithm. The OJF scheduling algorithm can only be applied to bipartite conflict graphs; therefore, we need to do this transformation first. Graph $\mathrm{G}$ in Fig. 2 can be transformed into a bipartite graph $\mathrm{G}_{-}$by merging vertices 1 and 2, 3 and 4, 5 and 6, and 7 and 8. Fig. 3 shows the bipartite graph. We describe the OJF scheduling algorithm as follows.

Algorithm 1: OJF scheduling algorithm.

Let ari , arj, alk, and alm be the earliest arrival times on each of the vertices of $\mathrm{G}_{-}$; while $r, r_{-}, 1,1$ have jobs waiting, do

Let ast be the earliest arrival time among ari , arj, alk, and alm;

Let $\mathrm{S}$ be the side of $\mathrm{G}_{-}$on which vertex s lies; for Each vertex s_on side $\mathrm{S}$ in $\mathrm{G}_{-}$, do

Schedule the job with the earliest arrival ast ;

Let $r$ and $r \_$be the vertices on the right side, and let 1 and $1 \_$be the vertices on the left side of the bipartite graph. Let $\mathrm{L}$ be the list of jobs that would arrive at the vertices in some time interval. Since we have no prior knowledge of the composition of L, the OJF algorithm aforementioned in Algorithm 1 makes decisions on the fly to reduce the maximum latency and is hence an online algorithm. For example, there exists an algorithm $A *$ that, given L, generates the optimal schedule (a schedule that minimizes maximum latency). A* is the optimal offline algorithm (see Table I for notations). Let us compare the performance of OJF and A* when it comes to minimizing the maximum latency. We claim that the OJF scheduling algorithm is 2-competitive, i.e., for any L, OJF produces a schedule where the maximum latency experienced by any job is at most twice the maximum latency experienced by any job in a schedule produced by A*. Thus, the OJF algorithm is 2-competitive. Furthermore, it turns out that there cannot exist a better than 2-competitive algorithm for job scheduling under the assump-tion of no future knowledge.

\section{B. OPTIMALITY OF THE OJF ALgORITHM}

Here, we prove that the algorithm presented earlier obtains the optimal competitive ratio. We adopt the proof for a bipartite graph with $\mathrm{n}$ vertices given in [17]. The method is based on an adversary technique in which the adversary creates a sequence of job arrivals based on the behavior of the online algorithm. At the beginning of each time unit, the adversary can determine how many jobs arrive and on which vertices of the conflict graph. After the entire job sequence has been determined, theadversary then can determine a schedule for the jobs in an offline manner. The adversary tries to create the worst sequence possible for the online scheduling algorithm. The cost of the online algorithm is then compared with the cost of the adversary determined offline algorithm. For a four-leg intersection, we consider to reproduce the same proof for the much easier case of $n=4$, which makes the proof shorter.

\section{VEHICULAR ADHOC NETWORK BASED TRAFFIC INTERSECTION CONTROL}

Here, we show how we implemented the platooning phase (phase one) of the OAF algorithm and how we implemented the other traffic light control schemes, such as the vehicle-actuated logic and Webster's method using VANETs. We first explain some of the terms used in describing our adaptive traffic control algorithms that may differ slightly from their conventional definitions.

- MAX-OUT: The maximum amount of GREEN time that can be allocated to the current phase.

- GAP-OUT: If a vehicle is more than the GAP-OUT units of time away from the stop line, then the signal goes to the next phase.

- EXTENSION: If a vehicle is detected less than GAP-OUT units of time away from the stop line, then the GREEN time is extended by EXTENSION units of time.

\section{A. SyStem Description}

In this paper, we study an isolated intersection. Fig. 1 shows the single traffic intersection under consideration. It is a typical four-leg intersection with eight traffic movement groups represented by the arrows. Each of the legs of the intersection is L meters long, and each of the left turning bays is B meters long. The numbered arrows show the directions of the various traffic movements. For this type of traffic intersection, we 
now describe the system architecture of the VANET-based traffic signal controller. In the single traffic intersection scenario, the traffic signal controller is connected to a wireless receiver that is placed at the intersection. The wireless receiver listens to information being broadcast from the vehicles. The broadcast medium is the 5.9-5.95-GHz radio spectrum, and the communication standards are defined in the IEEE $802.11 \mathrm{p}$ standards [19]. This system architecture is shown in Fig. 4. The information consists of speed and position data collected from vehicles. Speed data can be gathered from the vehicle speedometers, and position data can be gathered using GPS receivers fitted to the vehicles. In our implementation, the following data are gathered and encapsulated in data packets that are broadcast over the wireless medium. This is what we call the data dissemination phase.

Vehicle ID: Every vehicle is uniquely identified by its Vehicle ID\#. In practice, the medium access control (MAC) address of the network interface card in the wireless receiver would serve the same purpose.

Location: each vehicle is speci-fied by the LINK NUMBER\#, Lane\#, and position from a point of reference. The position from a point of ref-erence is a subfield containing $(\mathrm{x}, \mathrm{y})$, which are floating point quantities. We chose to use the stop line as a point of reference; therefore, the stop line has position $(0,0)$ for each Link Number\# and Lane\#. Thus, collectively, these three fields describe vehicle location. In practice, it is assumed that each vehicle is equipped with a GPS receiver; therefore, vehicles always know their locations. It is possible to convert the GPS coordinates of each vehicle to the format that we described earlier. We will show later that we can compute the distance from each vehicle to the stop line from this information.

Speed: Speed of a vehicle is a floating point quantity expressed in meters per second and is obtained from the in-vehicle speedometer sensor.

Current Time: The time at which the packet was created. The format is (hh:mm:ss). Because of the nature of the traffic control application, there is no need for a finer grain time. However, we need to assume that all clocks are synchronized. The current time is required to distinguish between old packets and new packets.

After the data dissemination phase, we have the data aggre-gation and processing phase where we actually make use of the transmitted information to do traffic signal control.

\section{B. Platooning Algorithm}

We obtained the lower bounds on how well an online algorithm can perform when it comes to minimizing the maximum latency. These lower bounds were achieved by an online algorithm that had no knowledge of future inputs. Can we use information gathered from the VANET to obtain future knowledge of traffic and use this to obtain a better-than-2-competitive algorithm? Unfortunately, this is unlikely; since due to radio range limitations, the VANET can only provide a relatively myopic view of the future, and in the long run, we will fall back to a 2-competitive performance. However, we can use the information from the VANET in a different way. One of the conditions under which the performance bounds hold is that all jobs, which represent platoons of vehicles, are of equal size and hence require equal processing time. This means that, for the OJF algorithm to be effective, all platoons must require equal amounts of time to pass through the intersection. We can achieve this requirement by using the vehicle position and speed data obtained via the VANET to compute the spatial headways between the vehicles. We can then divide the vehi-cles into platoons using this headway information, where each platoon takes equal amount of time to pass through the intersec-tion. This platooning phase will be the phase one of the OAF algorithm, with the OJF being phase two of the OAF algorithm.

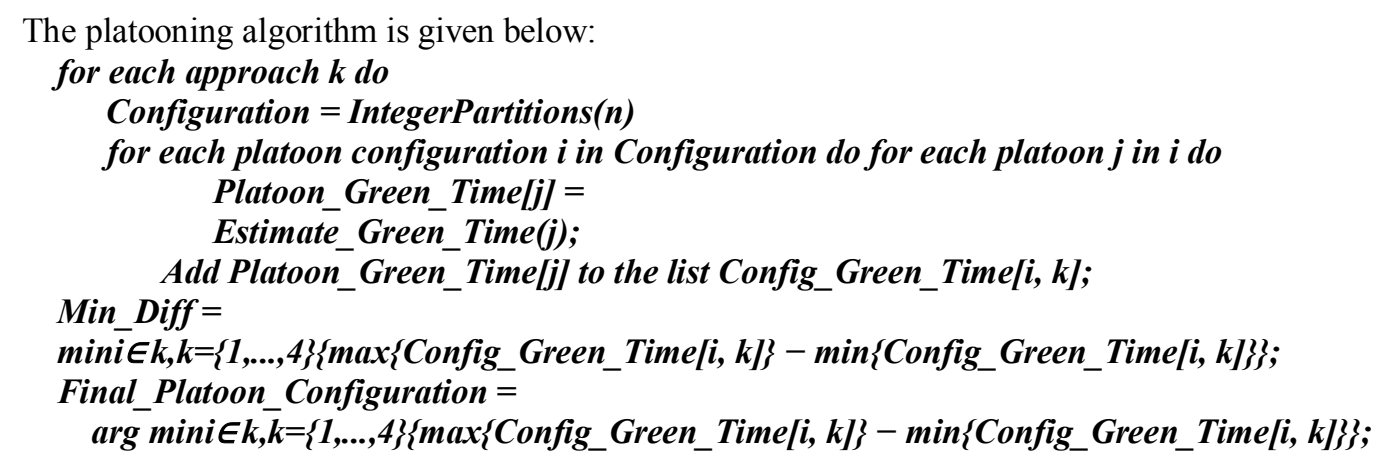




\section{RESULTS AND DISCUSSION}

We compare the performance of the OAF algorithm against the VANET-enabled vehicle-actuated control, VANET-enabled Webster's method, and an optimized fixed-time signal control. For the fixed-time approach, the controller has been optimized for the current traffic parameters, following the guidelines in [7]. The timing parameters were $60 \mathrm{~s}$ of GREEN for the through traffic and $30 \mathrm{~s}$ of GREEN for the left turning for the heavy traffic condition, $40 \mathrm{~s}$ of GREEN for the through traffic and $20 \mathrm{~s}$ of GREEN for the left turning traffic for the Medium traffic condition, and $35 \mathrm{~s}$ of GREEN for the left turning traffic for the Medium traffic condition, and $35 \mathrm{~s}$ of GREEN for the through traffic and $15 \mathrm{~s}$ of GREEN for left turning traffic for the light traffic. We also tested the effectiveness of the platooning algorithm, which is part of the OAF signal control algorithm.

\section{A. Comparison of Traffic Signal Control Methods During Homogeneous Traffic Arrival RATES}

In our first experiment, we have homogeneous traffic arrival rate on all four approaches to the intersection, and we vary the traffic arrival rates.. 7 shows the performance of the OAF in comparison with the vehicle-actuated logic, the pretimed logic, and the Webster's logic. The performance parameter that we measured was the average delay per vehicle in terms of seconds, and we plot this delay value at 5-min intervals for all the traffic signal control methods.

The labels light, medium, and heavy indicate the time in-tervals during the simulation with different traffic arrival rates. We started out the simulation at a light traffic arrival rate, and 20 min into the simulation, we switched to the medium traffic arrival rate. At the 40-min mark, we switched to a heavy arrival rate, and at 75 min mark, we switched to the light traffic arrival rate, and let the simulation run to the 160 -min mark. We can see that, at every instant, the OAF Algorithm performs better than both Webster's and the pretimed algorithm. During a heavy traffic arrival rate, we see that the OAF algorithm degenerates to the vehicle-actuated control since the MAX-OUT times for both the OAF algorithm and the vehicle-actuated algorithm become the same. However, when we switch back to the Light traffic arrival rate, we see that the OAF Algorithm recovers from congestion much faster; hence, the delays experienced decreased much faster. This is because the OAF algorithm is able to take advantage of the gaps that occur among vehicles and create platoons, and then, it minimizes the maximum delay that each platoon experiences.

The delay is then amortized among all the vehicles in the platoon. In effect, it is much more efficient at discharging the queues. Intuitively, this is because Webster's method and the pretimed control method both react very slowly to the changes in the traffic arrival rate.

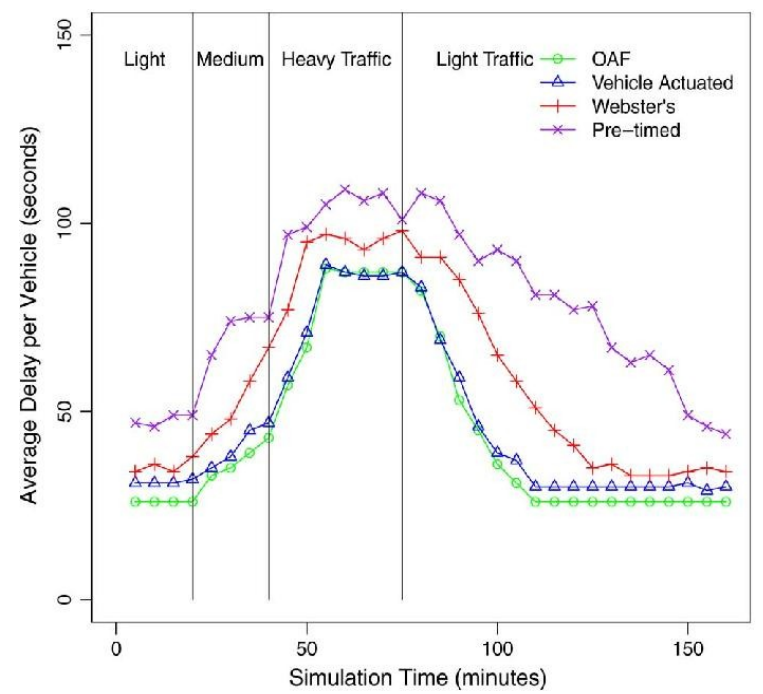

Fig. 7. Performance of OAF algorithm compared with other VANET-based traffic signal scheduling methods when all four approaches have equal vehicle arrival rates

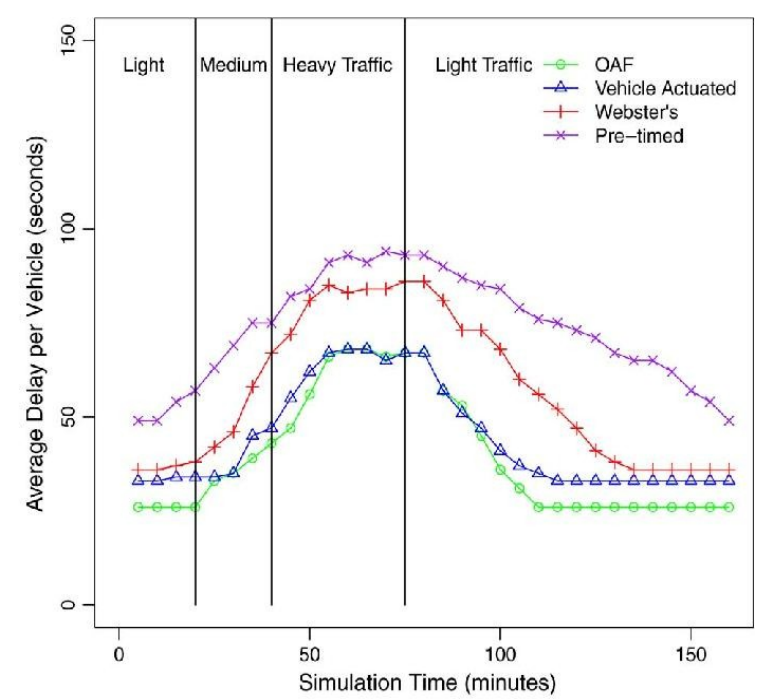

Fig. 8. Performance of the OAF algorithm compared with other VANET-based traffic signal scheduling methods when northsouth approaches have constant $(800$ vehicles/hour) arrival rates and east-west approaches have a varying vehicle arrival rate. 


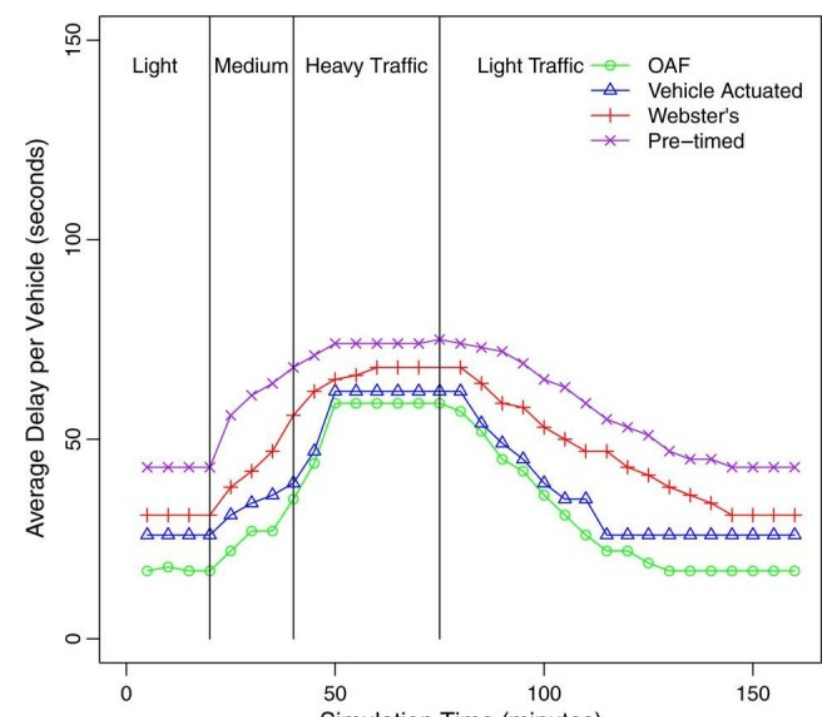

Fig. 9. Performance of the OAF algorithm compared with (minutes) ${ }_{\text {Ohther }}$ VANET-based traffic signal scheduling methods when north-south approaches have constant (100 vehicles/hour) arrival rates and when the east-west approaches have a varying vehicle arrival rate.

\section{B. Comparison of Traffic Signal Control Methods for Heterogeneous Traffic Arrival RATES}

Next, we study the performance of the OAF and the other three traffic control algorithms for the case with heterogeneous traffic arrivals. We set up the experiment in the following way. In Figs. 8 and 9, the east-towest traffic and the west-to-east traffic are set at 800 and 100 vehicles/h, respectively, but the north-to-south traffic and the south-to-north traffic vary from 400 (light), 800 (medium), and 1700 (heavy) vehicles/h and then back to 400 (light) vehicles/h.

Once again, there is a 0.15 probability that a vehicle makes a left turn. Once again, we compare the average delay per vehicle of the OAF algorithm and the vehicle-actuated traffic control algorithm against the Webster's and pretimed traffic signal control methods. Again, the OAF outperforms all the other three algorithms. Because of the lower variance in the traffic arrival rates, the delay curves are flatter than in the identical traffic arrival rate experiment, and the overall delays are lower for all the traffic signal control methods. The OAF and vehicle-actuated traffic control algorithms perform better than both Webster's method and the pretimed logic. However, because of the slower variance of the traffic arrival rates, the vehicle-actuated traffic control algorithm exhibits a flatter average delay curve.

\section{CONCLUSION}

In this paper, we have shown how a VANET can be used to aid in traffic signal control, including a new job-scheduling-based online algorithm, i.e., the OAF algorithm. We imple-mented several adaptive traffic signal control algorithms that use the fine grain information broadcasts by the vehicles. We implemented and compared these algorithms under various traffic conditions. Our experimental results show that the OAF algorithm reduces the delays experienced by the vehicles as they pass through the intersection, as compared with the other three methods under light and medium vehicular traffic loads. Under heavy vehicular traffic load, the performance of the OAF algorithm degenerates to that of the vehicle-actuated traffic method but still produces lower delays, compared with Webster's method and the pretimed signal control method. This is because, under lighter traffic, the OAF algorithm can dynamically skip through phases and minimize the delay of vehicles whenever there is a gap in the traffic. However, when the traffic gets heavier, the gaps in traffic disappear, and we always have queues on the approaches, reducing the advantage that a dynamic scheduling algorithm may have.

\section{FUTURE WORK}

Consider the case of sub lanes connected with main lanes. The vehicle which are at the head of platoon sends information to the vehicles near to sub lanes indicating that GREEN signal is ON in V2V communication. It helps the vehicles to use main lanes itself rather than using sub lanes. 


\section{REFERENCES}

[1]. G. F. Newell, Theory of Highway Traffic Signals, 6th ed. Berkeley, CA, USA: Univ. California, 1989.

[2]. D. C. Gazis, Traffic Science, 1st ed. New York, NY, USA: Wiley, 1989.

[3]. Optimal Traffic Control: Urban Intersections, 1st ed. Boca Raton, FL, USA: CRC, 2008, pp. 400-401.

[4]. C. N. Chuah, D. Ghosal, A. Chen, B. Khorashadi, and M. Zhang, "Smoothing vehicular traffic flow using vehicular_based ad hoc network-ing amp; computing grid (VGrid)," in Proc. IEEE ITSC, Sep. 2006, pp. 349-354

[5]. V. Gradinescu, C. Gorgorin, R. Diaconescu, V. Cristea, and L. Iftode, “Adaptive traffic lights using car-to-car communication,” in Proc. IEEE65th VTC-Spring, Apr. 2007, pp. 21-25.

[6]. N.Hounsell, J. Landles, R. D. Bretherton, and K Gardener, "Intelli- systems for priority at traffic signals in London: The INCOMEP roject," in Proc. 9th Int. Conf. Road Transp. Inf. Control, Number 454, 1998, pp. 90-94.

[7]. S. Irani and V. Leung, "Scheduling with conflicts," in Proc. 7th Annu. ACM-SIAM SODA, Soc. Ind. Appl. Math, Philadelphia, PA, USA, 1996, pp. 85-94.

[8]. D. Jiang and L. Delgrossi, "Ieee 802.11p: Towards an international stan-dard for wireless access in vehicular environments," in Proc.IEEE VTC Spring, May 2008, 2036-2040.

[9]. C. Priemer and B. Friedrich, "A decentralized adaptive traffic signal control using v2I communication data," in Proc. 12th Int. IEEE ITSC,Oct. 2009, pp. 1-6.

[10]. S. Phillips, R. Motwani, and E. Torng, "Non-clairvoyant scheduling,"in Proc. 4th Annu. ACM- SIAM SODA, Soc. Ind. Appl. Math.,Philadelphia, PA, USA, 1993, pp. 422-431.

[11]. S. G. Shelby, "Design and evaluation of real-time adaptive traffic sig-nal control aalgorithms," Ph.D. dissertation, Univ. Arizona, Tucson, AZ, USA, 2001. 